\title{
Soft Supersymmetry Breaking and the Supersymmetric Standard Model*
}

\author{
Savas Dimopoulos ${ }^{\mathrm{a}}$ \\ aPhysics Department, Stanford University, Stanford, CA 94305-4060, USA.
}

We recall how the idea of Softly Broken Supersymmetry led to the construction of the Supersymmetric Standard Model in 1981. Its first prediction, the supersymmetric unification of gauge couplings, was conclusively verified by the LEP and SLC experiments 10 years later. Its other predictions include: the existence of superparticles at the electroweak scale; a stable lightest superparticle (LSP) with a mass of $\sim 100 \mathrm{GeV}$, anticipated to be a neutral electroweak gaugino; the universality of scalar and gaugino masses at the unification scale. The original motivation for the model, solving the hierarchy problem, indicates that the superparticles should be discovered at the LHC or the TeVatron.

\section{Introduction}

It is a pleasure to recall the ideas that led to the Supersymmetric Standard Model. Supersymmetry is a marvelous theoretical idea whose mathematical foundations originate in the early the '70s[1,2]. In spite of this, it took a decade before a potentially realistic theory, one that is not obviously wrong, was proposed in 1981. The basic ingredient, missing until that time, was the concept of Softly Broken Supersymmetry. This is analogous to the history of the standard model whose mathematical foundations were laid down by Yang and Mills in the '50s, but whose development had to wait until the '60s; the missing idea in that case was that of spontaneous symmetry breaking.

In this talk I will mostly concentrate on my papers with Howard Georgi [3] from the spring of 1981 in which the idea of Soft Supersymmetry Breaking was proposed and used to construct what is now called the Supersymmetric Standard Model (SSM)as well as its unification into $S U(5)$ [4. The catalyst for our work was the hierarchy problem [5.6]. At present, the main reason why the unified Supersymmetric Standard Model enjoys its status as the leading contender for physics beyond the standard model is a quantitative pre-

\footnotetext{
*Invited talk presented at the "Thirty Years of Supersymmetry" Symposium, University of Minnesota, October 1315,2000 .
}

diction, dating from this paper, that has been verified by high precision data: that is a correlation between $\alpha_{s}\left(M_{Z}\right)$ and $\sin ^{2}\left(\theta_{W}\right)$ which has been confirmed by experiment at the $1 \%$ level [7] and shows that in the presence of superparticles at around a $\mathrm{TeV}$ the gauge forces of nature unify at a scale of $\sim 2 \times 10^{16} \mathrm{GeV}$. In fact this is the only significant quantitative success of any extension of the standard model and -together with neutrino masses- is the strongest experimental indication for new physics. The success of this prediction depends crucially on having both Unification and low energy Supersymmetry in the same theory; either Unification or Supersymmetry alone are insufficient. So, although we have not seen any real superparticles yet, we have evidence for Supersymmetric Unification via the effects of virtual superparticles running around loops at energies between the weak and the unification scale!

We present the developments in chronological order, beginning with an overview of the status of model building before 1981 (section 2). In section 3 we present the early work with S. Raby and F. Wilczek on the supersymmetric unification scale and the absence of proton decay in supersymmetric theories. Section 4 focuses on the papers with H. Georgi where we introduced the soft terms and the basic ingredients of the supersymmetric standard model, including the supersymmetric unification prediction. Section 5 deals with some of the important theoretical developments that 
followed. Section 6 discusses the significance of the unification prediction and its implications for model building and string theory. We end with an evaluation of the present status of the Supersymmetric Standard Model in section 7.

\section{Before 1981.}

Hierarchy Problem: A crucial turning point in our field occurred in the Spring of 1978. The SLAC experiment on parity violation in neutral currents convinced many theorists that the Standard Model of Glashow, Weinberg and Salam was correct and that it was a good time to start focusing on the next layer of questions: to explain some of the puzzling features of the Standard Model. The first question that theorists turned to was the "hierarchy problem" [5]: attempting to understand why the Higgs mass is so much smaller than the Planck mass or the Unification Scale. The Higgs does not carry any symmetry that ensures its lightness; indeed, in the absence of miraculous cancellations, the Higgs mass would be driven to the Planck or unification scale; it would not be available at low energies to do its intended job of giving mass to the weak gauge bosons and fermions.

Susskind and Weinberg [9] proposed the very appealing idea of Technicolor, as an alternative to the Higgs, for giving mass to the weak gauge bosons. In early '79 Technicolor was enlarged into "Extended Technicolor" 10 to allow the quarks and leptons to get their masses. By the summer of 1980 it became clear that these theories suffered from generic problems of flavor violations [11] that could perhaps be cured only by complicating the theory immensely and losing any hope of calculability. I, perhaps prematurely, felt that this was too high a price to pay and decided to look at other alternative approaches to the Hierarchy problem.

That is when we turned to Supersymmetry [1.22]. It was generally realized that Supersymmetry could help the hierarchy problem [6]. The reason is that the Higgs, a scalar, would form a degenerate pair with a fermion, called the Higgsino. Since the Higgsino could be protected by a chiral symmetry from becoming superheavy, so could its degenerate scalar partner, the Higgs. Of course Supersymmetry does much more than to just relate the Higgs to the Higgsino. It assigns a degenerate scalar "superpartner" to each and every known quark and lepton, as well as a fermionic degenerate superpartner to each gauge boson. Since no such particles had been seen it was clear that Supersymmetry had to be a broken symmetry. Nevertheless, Supersymmetry would still help the hierarchy problem as long as its breaking occurs near the weak scale. This had the immediate implication that the superpartners had to be at accessible energies! This line of reasoning led us to begin our attempt to find a Supersymmetric version of the Standard Model with Supersymmetry broken at the weak scale. Together with Stuart Raby and Leonard Susskind we started learning about Supersymmetry and tried to find out if such theories had already been constructed. We quickly discovered that no Supersymmetric versions of the Standard Model existed at that time.

Broken Charge and Color: There were early attempts by Fayet 12 to build models where supersymmetry was broken spontaneously in the standard model sector. They were all plagued by a plethora of problems including: the breaking of electromagnetic gauge invariance, predicting a photon mass $\sim 100 \mathrm{GeV}$; the breaking of color symmetry at the electroweak scale; massless gluinos, a consequence of the problematic continuous R-symmetry of these models. Attempts to cure these problems by enlarging the gauge group to led to anomalies whose cure again led to the breaking of the electromagnetic and color gauge invariance. The root of these problems was that in these theories Supersymmetry was broken spontaneously at the tree level. In 1979 a very important paper by Ferrara, Girardello and Palumbo 13 showed that in such theories, under very general conditions, color and charged scalars would get negative masses squared, leading to breaking of electric charge and color. This essentially stopped efforts to build realistic Supersymmetric theories. It was hard to take seriously theories in which photons and gluons weighed $\sim 100 \mathrm{GeV}$.

Supercolor: We spent early fall of 1980 rediscovering these problems. It rapidly became clear 
that the breaking of supersymmetry had to originate outside the standard model. Our first attempt was to break Supersymmetry dynamically with a new strong force, very similar to Technicolor, which we called Supercolor. We were not alone in these efforts. Witten [6] as well as Dine, Fischler and Srednicki [6] were pursuing similar ideas for precisely the same reasons. They wrote two very important papers entitled "Dynamical breaking of Supersymmetry "(Witten) and "Supersymmetric Technicolor" (Dine, Fischler and Srednicki). Their preprints appeared in April of '81 at the same time as our "Supercolor" paper [6].

An essential objective of these works was to point out that low energy Supersymmetry helps the hierarchy problem 9 , and to argue that a new strong force analogous to QCD or Technicolor may induce the breaking of Supersymmetry and explain the smallness of the electroweak scale. Dine, Fischler and Srednicki, as well as Raby and myself, also attempted to build explicit models incorporating these ideas, but without much success. I do not have time to discuss these "Supercolor" or "Supersymmetric Technicolor" theories. They had problems; one of them was that they were baroque. By January of 1981 we were discouraged. Although Stuart Raby and I had begun writing the Supercolor paper [6], we already did not believe in it. It seemed too much to believe that Nature would make simultaneous use of Supersymmetry and Technicolor to solve the hierarchy problem.

\section{3. "Supersymmetry and the Scale of Uni- fication."}

In spite of these obstacles, we were mostly optimistic that the problem of supersymmetry breaking would eventually be solved. In the meantime we were getting anxious to start doing physics with the idea of weak-scale supersymmetry. A result of this was the early paper with Stuart Raby and Frank Wilczek [14 in which we computed the Unification Mass in the presence of the mini-

\footnotetext{
${ }^{2}$ Lots of people, in addition to those in Reference [6], were aware of this. The challenge was to implement the idea in a consistent theory with weak-scale Supersymmetry .
}

mal Supersymmetric particle content at the weak scale. We found that, because the superpartners of the gauge bosons slow down the evolution of the couplings, the unification mass increased to about $10^{18} \mathrm{GeV}$. This was interesting for two reasons:

- This value is close to the Planck mass, perhaps suggesting eventual unity with gravity. This connection subsequently got weaker as more accurate calculations [3,7,76] reduced the value to $\sim 2 \times 10^{16} \mathrm{GeV}$.

- There was a distinct experimental difference with ordinary $S U(5)$ : the proton lifetime was unobservably long.

The latter appeared to be an easily disprovable prediction. In fact by that time three different experimental groups had reported preliminary proton decay "candidate events": the Kolar gold field, Homestake mine and the Witwatersrand experiments. We knew that S.Miyake, of the Kolar Gold Field experiment, and possibly representatives of the other experiments were going to talk about their events in the upcoming "Second Workshop on Grand Unification" where I was also going to present our theoretical results [8]. So, I was a bit nervous but did not hesitate to present them. I am proud of this paper: A simple and well motivated ingredient, virtual $\mathrm{TeV}$ superparticles, made a big difference to a quantity that was being measured at that time, the proton lifetime. Perhaps this is the first test that supersymmetric unified theories (SUSY-GUTs) have passed. In this paper, although we pointed out that the value of $\sin ^{2}\left(\theta_{W}\right)$ would change due to the Higgs sparticles, we did not present the new value. After satisfying ourselves that it would not be grossly modified, we focused on the change in the unification mass, which at that time was more important for experiment.

The next big step was to construct a realistic supersymmetric theory.

\section{4. "Softly Broken Supersymmetry and SU(5)."}

Soft Supersymmetry Breaking: In the meantime, the problem of supersymmetry break- 
ing continued to be a major obstacle to building a realistic supersymmetric extension of the standard model. After finishing the previous paper we, in collaboration with Howard Georgi, returned to this problem. The prevailing view at that time was that a realistic Supersymmetric model would not be found until the problem of Supersymmetry Breaking was solved. It was further believed that the experimental consequences of Supersymmetric theories would strongly depend on the details of the mechanism of Supersymmetry breaking. After all, it was this mechanism that caused the phenomenological disasters of the early attempts.

The key that took us out of this dead end was the realization that a search for a detailed mechanism of supersymmetry breaking might be futile, unless it also solves the cosmological constant $(C C)$ problem. Any mechanism that fails to do this appears so massively wrong that it seems pointless to trust its secondary implications, such as its spectroscopy. This, admittedly idealistic view, led us to seek a far more general approach to supersymmetry breaking; one which would have a better chance of adapting to describe the effect on the standard model superparticles of the -still unknown- "correct supersymmetry breaking mechanism" which must solve the CC problem.

This thought naturally focused us on the standard model sector and led us to the simplest hypothesis: to start with a supersymmetric version of the standard model and just add all the terms which break supersymmetry "softly". Our definition of "softly" was dictated by our desire to address the hierarchy problem: it meant that supersymmetry breaking went away rapidly enough at high energies that it did not cause any quadratic divergences to the Higgs mass. The virtue of this simple effective-field-theoretic approach is that it is general enough to have a chance of adapting to the correct ultimate mechanism.

It has some immediate physics implications, since it implicitly postulates that the dynamics that breaks Supersymmetry is external to the ordinary $S U(3) \times S U(2) \times U(1)$ sector; specifically, it implies that:
1. The only particles carrying $S U(3) \times S U(2) \times$ $U(1)$ quantum numbers are the ordinary ones and their Superpartners that reside at the weak scale. Extra particles with exotic $S U(3) \times S U(2) \times U(1)$ quantum numbers are unnecessary. This is essential for the successful unification prediction.

2. Ordinary particles and their superpartners do not carry any extra new gauge interactions at low energies. This too is important for the unification prediction.

In summary, the successful gauge coupling unification is evidence in favor of these two implications of the hypothesis of soft supersymmetry breaking. The hypothesis postulates that the origin of susy breaking lies outside the standard model particles and therefore leaves the standard model degrees of freedom as simple as can be.

Main Results: The hypothesis of soft susy breaking immediately led to the two papers [3] entitled "Softly Broken Supersymmetry and SU(5)" and "Supersymmetric GUTs" which first proposed the supersymmetric standard model. More precisely, these papers accomplished three objectives:

1. Supersymmetric Unification (SUSYGUTs): Construction of a Unified supersymmetric theory of strong and electroweak forces. Our gauge group was $\mathrm{SU}(5)$. Unification was essential for the prediction of $\sin ^{2}\left(\theta_{W}\right)$ and for some of the phenomenology, such as proton decay and gaugino masses. It was also important for addressing the hierarchy problem and related issues such as doublet-triplet splitting.

2. Supersymmetry Breaking: Supersymmetry was broken softly by mass terms for all scalar superpartners and gauginos. The origin of supersymmetry breaking lay outside the standard model degrees of freedom, as explained earlier in this section. "Softly" ensured that the Higgs mass had no quadratic sensitivity on the unification mass. 
3. Supersymmetric Standard Model: As a bonus, our theory contained the first phenomenologically viable supersymmetric extension of the standard $S U(3) \times S U(2) \times$ $U(1)$ model (SSM), already imbedded inside the unified theory.

We constructed the model in late March and early April of 1981. We were very pleased. We had the first realistic Supersymmetric theory, incorporating all non-gravitational phenomena and valid up to the Planck mass. We immediately started thinking about experimental consequences. We wanted to make sure that we would not miss anything important. Time pressure helped us a lot. Both Howard and I were scheduled to give two consecutive talks in the Second Workshop on Grand Unification which took place at the University of Michigan on April 24-26, 1981 [8]. Here are some of our phenomenological results that we reported in that Workshop [3]:

- $\sin ^{2}\left(\theta_{W}\right)$ : We presented our SUSY-GUT prediction for $\sin ^{2}\left(\theta_{W}\right)$. The magnitude we got disagreed with the then central experimental value, but the errors were large. We argued that there would have to be 2 Higgs doublets for the value not to be way off.

- Proton Decay: We reported that the Supersymmetric Unification Mass is so large 14] that proton decay is unobservably small.

- Superparticle Spectroscopy: squarks and sleptons. We noted that if all squarks and sleptons have a common universal mass $\left(\sim M_{W}\right)$ at the unification scale, there would be a "Super-GIM mechanism" supressing neutral flavor violations. The Higgses could have different masses.

- Superparticle Spectroscopy: gauginos. Because we had a unified theory all gauginos had a common Majorana mass $\left(\sim M_{W}\right)$ at the unification scale.

- Family Reflection Symmetry; Stable LSP. To avoid rapid proton decay via dimension-four operators we postu- lated a discrete symmetry forbidding threefamily couplings. This symmetry was subsequently called family reflection symmetry 18] or matter parity 3 . We concluded:

"the lightest of the supersymmetric particles is stable. The others decay into it plus ordinary particles. One simple possibility is that it is the U(1) gauge fermion."

It is gratifying that the above ingredients have survived the test of time. They form the basis of what is now called the minimal supersymmetric standard model (MSSM). Perhaps the most important conclusion of our paper is also the one that now seems so evident because it has, with time, been incorporated into our thinking:

"The phenomenology of the model is simple. In addition to the usual light matter fermions, gauge bosons and Higgs bosons, we predict heavy matter bosons, gauge fermions and Higgs fermions as supersymmetric partners. We can say little about their mass except that they cannot be very large relative to $1 \mathrm{TeV}$ or the motivation for the model disappears." [3]

Of course, our motivation was to address the hierarchy problem; without it we could not have drawn this conclusion.

Early Reception: Georgi and I spoke on the last day of the conference [8]. My feeling then was that our results were for the most part ignored, especially by the experimentalists who did not care about the hierarchy problem. Our conclusions were very much against the spirit of the conference. There were three things against us:

(1)The central value of the weak mixing angle agreed better with the predictions of ordinary (non-Supersymmetric) Grand Unified Theories, albeit with large error bars.

(2) Preliminary proton decay "candidate events" had been reported by three different experimental groups, the Kolar gold field, Homestake mine and the Witwatersrand experiments.

${ }^{3}$ It turned out to be equivalent to a discrefe subgroup of the problematic continuous R-symmetry 12 . 
(3)The host institution was gearing up to launch the then biggest effort on proton-decay, namely the IMB experiment.

The atmosphere in the conference is summarized by Marciano's April 24, 1981 concluding remarks [8]:

"The basic idea of Grand Unification is very appealing. The simplest model based on $S U(5)$ has scored an important success in predicting a value for $\sin ^{2}\left(\theta_{W}\right)$ which is in excellent agreement with recent experimental findings (after radiative corrections are included). It makes an additional dramatic prediction that the proton will decay with a lifetime in the range of $10^{30}-10^{32}$ years. If correct, such decays will be seen by the planned experiments within the coming year (or may have already been seen). An incredible discovery may be awaiting us."

In spite of this, theorists that cared about the hierarchy problem were pleased with our work. This included Sheldon Glashow, Leonard Susskind and Steven Weinberg. In his April 26, 1981 conference summary talk [8] Weinberg mentioned our theory and its predictions of $\sin ^{2}\left(\theta_{W}\right)$ and $M_{G U T}$ several times. Weinberg's verdict [8]:

"...the model of Dimopoulos and Georgi has many other attractive features and something like it may turn out to be right."

This was music to my ears. In May I presented our results in two more conferences, one in Santa Barbara and the other at the Royal Society in London. Soon afterwards theoretical activity in supersymmetric unification began to pick up. In August of '81 Girardello and Grisaru wrote a very important paper [15] systematically discussing explicit soft breaking of global supersymmetry; they were the first to discuss cubic soft terms. Starting in July of ' 81 several important papers 16] repeated our calculation of the superunified value of $M_{G U T}$ and $\sin ^{2}\left(\theta_{W}\right)$, some improving it to two loops. Sakai's paper [16] also repeated our analysis of SU(5) breaking; it did not discuss the soft supersymmetry breaking terms

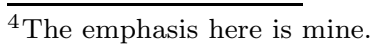

and thus did not address the spectroscopy and phenomenology of superparticles.

The interest in GUTs and SUSY-GUTs dwindled after 1983. The rise of superstrings, the absence of proton decay and the lack of precise data on $\sin ^{2}\left(\theta_{W}\right)$ were some of the reasons. The best evidence that the morale among the non-stringers was low is that the annual series of "Workshops on Grand Unification" was terminated. 1989 was the year of the "Last Workshop on Grand Unification". In the introduction to that terminal volume Paul Frampton exclaimed:

"Alas, none of the principal predictions of GUTs have been confirmed."

This was written in August 1989, just as LEP was beginning to take data...

\section{Completing the Picture.}

Since time is so short I have limited myself to those aspects of superunified theories that are least model-dependent and experimentally testable or, in the case of $\sin ^{2}\left(\theta_{W}\right)$ and proton decay, perhaps already tested. Of course, the theory that we proposed left some important theoretical questions unanswered. I will briefly mention some of the problems and related ideas.

Proton Decay Revisited: Although Georgi and I worried a lot about dimension-four baryon violating operators and we introduced the family reflection symmetry to forbid them, it did not occur to us to check the operators of dimension five! Weinberg 17] as well as Sakai and Yanagida [17] studied these operators and concluded that they pose a severe problem for our theory. They attempted to construct models with an extra $U(1)^{\prime}$ gauge group that would forbid the dimension five operators that mediated proton decay. Raby, Wilczek and I studied these operators in October of ' 81 and concluded that the small Yukawa couplings of the light generation naturally supressed them [18]. The resulting proton decay rates, although not calculable from low energy physics parameters, could be experimentally observable. Furthermore they had a very unique signature that is not expected in non-supersymmetric theories: protons and neu- 
trons decay into kaons. We were very excited that we had identified another "smoking gun" for supersymmetry. Ellis, Nanopoulos and Rudaz independently reached the same conclusions [18].

Doublet-triplet splitting: There is one remaining technically natural fine tuning in our theory [3]. Wilczek and I addressed this problem in June of 1981 and found two solutions now called the missing partner and the missing VEV mechanisms 19]. Attempts to implement these mechanisms in realistic theories led to complicated constructions 20.

Hidden sector: The theoretical question of how supersymmetry is broken and superparticle masses are generated in our theory attracted a lot of attention. Georgi and I had decided that, in the absence of a solution to the cosmological constant problem, any specific supersymmetry breaking mechanism was suspect and should not be relied upon to predict sparticle masses etc. This was a reason we proposed our more general softterms approach. Nevertheless, it was important to present at least an existence proof of a mechanism that generated our soft terms. An important consideration was that squarks and sleptons belonging to different generations had to have identical masses to avoid problems with rare processes [3]. In the winter/spring of ' 82 three different groups [21], Dine and Fischler, Raby and I, and Polchinski and Susskind came up with the idea of a Hidden Sector, around $10^{11} \mathrm{GeV}$, where supersymmetry breaking originates and is subsequently communicated to the ordinary particles via a new gauge interaction at the unification scale. Soon afterwards a series of very important papers developed a better idea for such a mechanism: $\mathrm{Su}-$ persymmetry breaking could be communicated from the hidden sector via supergravity [23].

Radiative electroweak breaking: Hidden sector mechanisms for Supersymmetry breaking, under very special assumptions, give degenerate masses to all scalars: squarks, sleptons as well as Higgses. This is good for avoiding flavor violations 3 but poses the puzzle: what distinguishes the Higgs from the squarks and the sleptons?

\footnotetext{
${ }^{5}$ For Raby and me the starting point was trying to build a realistic model utilizing Witten's idea of "Inverted Hierarchy" 22.
}

Why does the Higgs get a vacuum expectation value and not the squarks? Starting with Ibañez and Ross, a series of very important papers 24 developed the idea of radiative electroweak breaking which answers this question dynamically provided the top quark is sufficiently heavy, above $\sim 60 \mathrm{GeV}$.

The title of this section is misleading. The picture is still far from complete; many fundamental questions remain unanswered. The theory we have is definitely not a theory of everything. Instead, it is a phenomenological, disprovable theory that allows us to make contact with experiment in spite of the questions that it fails to address.

\section{How Significant is the Unification Pre- diction?}

Since the LEP data confirmed the SUSY-GUT prediction this topic has received a lot of attention and is discussed in many papers. My analysis will be somewhat outdated, based on the excellent analysis of Ref. [7] and the overview of ref [25]. The results have not changed much since then and supersymmetric unification continues to be successful. The estimated uncertainties in the theoretical predictions for SUSY-GUTs and GUTs are due to: $\alpha_{s}\left(M_{Z}\right)$ and $\alpha\left(M_{Z}\right)$ error bars, sparticle thresholds, $m_{t}$ and $m_{h^{0}}$, GUT thresholds and Non-renormalizable operators at the unification scale. For the $\sin ^{2}\left(\theta_{W}\right)$ prediction they all add up to about $\pm 1 \%$ [7] . The experimental error is negligible, $\pm 0.2 \%$. Experiment and theory agree and the probability that the agreement is an accident is $\sim 2 \%$. The largest source of theoretical uncertainty is due to the $\alpha_{s}\left(M_{Z}\right)$ error bar; this should shrink in the future. The other uncertainties are significantly smaller. The threshold corrections are proportional to $\alpha$ s times logarithms of mass ratios. For example, the total of the low energy sparticles' contributions is summarized in

\footnotetext{
${ }^{6}$ In the original SUSY-GUT this was not an issue because the Higgs masses were assumed to be different from the universal squark and slepton masses [3].

${ }^{7} \sin ^{2}\left(\theta_{W}\right)$ is in the $\overline{\mathrm{MS}}$ scheme.
} 
the following expression [7,26]:

$$
\begin{aligned}
\sin ^{2} \theta\left(M_{Z}\right) & =0.2027+\frac{0.00365}{\alpha_{3}\left(M_{Z}\right)} \\
& -\frac{19 \alpha_{e m}\left(M_{Z}\right)}{60 \pi} \ln \left(\frac{T_{S U S Y}}{M_{Z}}\right)
\end{aligned}
$$

where 8 ,

$$
\begin{aligned}
T_{S U S Y}= & m_{\widetilde{H}}\left(\frac{m_{\widetilde{W}}}{m_{\tilde{g}}}\right)^{28 / 19}\left(\frac{m_{\tilde{l}}}{m_{\tilde{q}}}\right)^{3 / 19} \times \\
& \times\left(\frac{m_{H}}{m_{\widetilde{H}}}\right)^{3 / 19}\left(\frac{m_{\widetilde{W}}}{m_{\widetilde{H}}}\right)^{4 / 19} .
\end{aligned}
$$

and $m_{\tilde{q}}, m_{\tilde{g}}, m_{\tilde{l}}, m_{\widetilde{W}}, m_{\widetilde{H}}$ and $m_{H}$ are the characteristic masses of the squarks, gluinos, sleptons, electroweak gauginos, Higgsinos and the heavy Higgs doublet, respectively. $T_{S U S Y}$ is an effective SUSY threshold.

From these equations we learn that the supersymmetric threshold corrections are typically small. The same holds for the high energy threshold corrections in minimal SUSY-GUTs [7]. Therefore the $\sin ^{2} \theta\left(M_{Z}\right)$ prediction is quite insensitive to the details of both the low and the high mass-scale physics; it takes a large number of highly split multiplets to change it appreciably. For example, we know that to bring $\sin ^{2} \theta\left(M_{Z}\right)$ down by just $\sim 10 \%$ - back to the standard $\mathrm{SU}(5)$ value - we would need to lift the higgsinos and the second higgs to $\sim 10^{14} \mathrm{GeV}$.

The flip side of these arguments show that to "fix" Standard non-supersymmetric GUTs, you also need several highly split multiplets [27. In fact you need many more than the supersymmetric case, since you do not have superpartners. In Standard GUTs either $\sin ^{2}\left(\theta_{W}\right)$ or $\alpha_{s}\left(M_{Z}\right)$ are off by many standard deviations. Worse yet, the proton decays too fast. Do these problems mean that all non-supersymmetric GUTs are excluded? Of course not. By adding many unobserved split particles at random to change the running of the couplings you can accommodate just about any values of $\sin ^{2}\left(\theta_{W}\right)$ and $M_{G U T}$. So, in what sense are these quantities predicted? I answer this with a quote from reference [28]:

${ }^{8}$ In eq.(2) if any mass is less than $M_{Z}$ it should be replaced by $M_{Z}$.
" Once we wander from the straight and narrow path of minimalism, infinitely many silly ways to go wrong lie open before us. In the absence of some additional idea, just adding unobserved particles at random to change the running of the couplings is almost sure to follow one of these. However there are a few ideas which do motivate definite extensions of the minimal model, and are sufficiently interesting that even their failure would be worth knowing about."9

\section{Peaceful Coexistence with Superstrings:}

The predictions of the heterotic string theory for $\sin ^{2}\left(\theta_{W}\right)$ (inputing $\alpha_{s}\left(M_{Z}\right)$ ) is off by 26 standard deviations 25]. Similarly, the prediction of $\alpha_{s}\left(M_{Z}\right)$ (inputing $\sin ^{2}\left(\theta_{W}\right)$ ) is off by 11 standard deviations. The reason is that in the heterotic models the string scale is rigidly connected to the observed value of the Planck mass and turns out to be a factor 20 bigger than the unification scale. As a result, in heterotic string theory, the predicted value of the proton mass is $20 \mathrm{GeV}$. The reaction of the string community to this disagreement was mixed. Many celebrated the indirect evidence for low energy supersymmetry as being "consistent with string theory". Some adopted the attitude that a discrepancy by a factor of 20 was not too bad, and chose to ignore that it was off by a large number of standard deviations. Others adopted the view that the success of the supersymmetric unification prediction was an accident and drew parallels between it and the near equality of the apparent size of the Sun and the Moon on the sky 10. Many found comfort in the possibility that very large threshold stringy corrections could be tuned to "fix" the problem. Of course, such a "fix" is no better than accommodating ordinary non-supersymmetric SU(5) with large corrections caused by random unobserved multiplets. The question remained 30]:

"why should these corrections maintain the relations between the couplings characteristic of the Grand Unified symmetry, if such a symmetry is

\footnotetext{
${ }^{9}$ Emphasis mine

${ }^{10}$ The success of supersymmetric unification is now taken more seriously and is the most common criticism of the large dimension framework 29 .
} 
not actually realised?"

This was the climate until a very important paper by Petr Horava and Edward Witten [31] took the supersymmetric unification prediction seriously and proposed to lower the string scale to match the SUSY-GUT scale of $\sim 10^{17} \mathrm{GeV}$. To explain the weakness of gravity they proposed a new class of 5-dimensional theories in which the relation between the string scale and the 4-dimensional Planck mass is not direct but involves the size of the 5th dimension. By choosing its size large enough, $\sim 10^{-28} \mathrm{~cm}$, one could account for the unification of gravity with the other forces at the now reduced string scale. Although it has not led to a realistic model, the scenario proposed by Horava and Witten is a good contemporary example of how input from experiment can help focus theoretical effort in a new direction.

\section{An Evaluation of the Supersymmetric Standard Model}

There is no question that the biggest success of the SSM is the unification of couplings. Since much of this talk has been devoted to that, we now want to discuss how well the SSM does with some other important phenomenological issues. Many of these are widely viewed as successes of the SSM and I will attempt to present a more balanced view of the pros and cons. The second virtue of the SSM - and its original motivation- is that it addresses the hierarchy problem, at least in the sense that it protects light scalars from ultraviolet physics. This is not quite the same as solving the hierarchy problem, which requires further dynamics for obtaining the weak mass from the GUT scale, but it is an ingredient ensuring the stability of the hierarchy. It is a definite plus, extensively discussed, and I have nothing to add. The remaining issues, often considered as virtues of the SSM are: proton longevity, dark matter LSP, neutrino masses, bottom-tau unification and approximate neutral flavor conservation. To start with, these are all qualitative and, as a result, less impressive than unification. We evaluate them in turn:

Proton Longevity: This is a virtue of the nonsupersymmetric and non-unified standard model, where the conservation of baryon and lepton numbers is an automatic consequence of gauge invariance. In contrast, in the supersymmetric theory we were forced to introduce an additional global symmetry, the family reflection symmetry, to account for the stability of the proton[3]. Such symmetries are also necessary in other extensions of the standard model, such as the large dimension framework 29]. In fact, the most recent SuperKamiokande limits to the proton lifetime are so severe that the dimension five operators of section 5 may be problematic for simple SUSY-GUTs. One has to either postulate that the color triplet Higgs-fermions are significantly heavier than the Planck mass or, more plausibly, that their vertices have a complicated flavor structure which comes to the rescue and suppresses the decay of the proton.

Dark Matter LSP: The existence of a stable lightest supersymmetric particle (LSP) as a dark matter candidate is a welcome qualitative feature of the SSM. Its stability is a consequence of the family-reflection-symmetry, postulated to account for the stability of the proton. This chain of reasoning - new physics at a $\mathrm{TeV}$ requires new symmetries to ensure a stable proton which in turn implies a new stable particle- is common. In the large dimension framework 29 there are several possibilities for stable DM candidates in the TeV range, such as matter on other walls or in the bulk. Furthermore, getting the correct abundance does not require a miracle. Stable particles in the TeV-range naturally have the right annihilation cross section to result in remnant abundance near closure density 32].

Neutrino Masses: The argument here is that the success of the seesaw mechanism is an indication for $S O(10)$-like physics at a large scale scale 33. Perhaps; but the actual scale associated with right handed neutrinos is significantly below the SUSY-GUT scale and the connection is one of rough orders of magnitude. Furthermore, an essentially identical -and equally looseconnection can be made in the large dimension framework 34. There, neutrino masses could be argued to give evidence for a large bulk!

Bottom-tau unification: This too is qualitative, and works about equally well in the non- 
supersymmetric standard model[35]. Furthermore, this relation fails for the lighter generations, perhaps because they are more susceptible to Planckean physics 36].

Approximate Neutral Flavor Conservation. This in fact is, just like proton decay, often interpreted backwards: We were forced to postulate the universality of scalar masses to account for the absence of neutral flavor violations [3]. One might like to argue in favor of this on grounds of simplicity. This is obviously not sufficient since there is no symmetry to ensure the universality of sparticles masses; the flavor symmetry is broken badly in the fermion sector and this breaking in general contaminates the scalar sector and creates unwanted large flavor violations [37], especially in the kaon system. The issue of how to avoid this is subtle and has sparked renewed interest in low-energy-gauge mediated theories [38]. There the problem of the contamination of soft terms by fermion masses is avoided because the soft supersymmetry breaking vanishes in the UV where flavor originates.

This is part of the challenging "Flavor Problem", one of the most serious for the SSM: that, even after we impose all the gauge symmetries (as well as the family-reflection global symmetry), the model has 125 parameters! [39]. Luckily, the vast majority of these parameters reside in the flavor sector of the theory and do not contaminate the successful prediction of the unification of gauge couplings.

In summary, the gauge sector of the SSM is compelling; the flavor sector requires care to ensure approximate flavor conservation and and proton stability. In contrast, the nonsupersymmetric unified theories [4] have problems in their gauge sector, both with respect to proton decay and gauge coupling unification.

An often unspoken practical virtue of the SSM is that it is a perturbative theory with detailed predictions, for any choice of parameters. Although this is not fundamental, it accounts for some of the popularity of the model. This is not the case for either technicolor or the large dimension framework, which eventually requires a full string theory model of the world at a $\sim \mathrm{TeV}$.

Of course, the most serious problem of the SSM is the cosmological constant $(\mathrm{CC})$ problem. It casts a dark shadow over everything, including the standard model. It is possible that all our efforts to go beyond the standard model based on the hierarchy problem are misguided, because they have nothing to say about the CC problem. On alternate days I think this is the right view and that looking under the hierarchy "lamp post" is leading us nowhere. The other days however I think that we can decouple the CC problem from the rest, perhaps because it involves gravity. Or, better yet, because Nature has already told us so, with the tremendous success of QED and the Standard model. Or, perhaps even by the very success of the supersymmetric picture of gauge coupling unification...

Because of my involvement with both the SSM and the large dimension idea, I am often asked "which do I believe is correct". Obviously, I am not more qualified than anybody else to answer this question. Still, the unification of coupling is more natural in the SSM and for this reason I have a preference for the SSM. However, as I tried to emphasize in this section, what we do not know far exceeds what we do. The normal desert picture has, for over $\sim 20$ years, failed to shed light on many questions, such as the flavor and the $\mathrm{CC}$ problems. For these reasons alone it seems worthwhile to consider alternatives that may provide a new perspective to old problems.

We are fortunate that in a few years experiment will tell us which road Nature chooses for breaking the electroweak symmetry. Either way, we will be living in exciting times. If it is supersymmetry will see the superpartners. If it is large dimensions we will see all of quantum gravity and string theory, so we will have an even more complete picture of the universe. Or perhaps, best of all, experiment will tell us something even more strange and exciting that none of us has dreamed.

\section{Acknowledgments}

I would like to thank Howard Georgi for sharing his recollections of the events that led us to the Supersymmetric Standard Model and for a careful reading of the manuscript. I would also like to thank: N. Arkani-Hamed, R. Barbieri, M.Carena, 
G.Giudice, N.Polonsky and C. Wagner for very valuable discussions; K. Olive, M. Shifman, S. Rudaz, A. Vainshtein and M. Voloshin for organizing a stimulating symposium and for their hospitality. My work, since my student years, has been supported by the National Science Foundation; present grant number: NSF-PHY-9870115003.

\section{REFERENCES}

1. Yu. A. Gol'fand and E.P. Likhtman, JETP Lett. 13 (1971) 323;

D.V. Volkov and V.P. Akulov, Phys. Lett. B 46 (1973) 109;

J. Wess and B. Zumino, Nucl. Phys. B 70 (1974) 39

2. D.Z. Freedman, P. van Nieuwenhuizen, and S. Ferrara, Phys. Rev. B 13 (1976) 3214;

S. Deser and B. Zumino, Phys. Lett. B 62 (1976) 335.

3. S. Dimopoulos and H. Georgi, "Supersymmetric GUTs", p 285, Second Workshop on Grand Unification, University of Michigan, Ann Arbor, April 24-26, 1981, eds. J.Leveille, L.Sulak, D.Unger; Birkhauser, 1981;

S. Dimopoulos and H. Georgi, Nucl. Phys. B 193 (1981) 150.

4. H. Georgi and S. Glashow, Phys. Rev. Lett. 327 (1974) 438;

J. Pati and A. Salam, Phys. Rev. D 8 (1973) 1240 ;

H. Georgi, H. Quinn and S. Weinberg, Phys. Rev. Lett. 33 (1974) 451.

5. K. Wilson, as mentioned in L. Susskind, Phys. Rev. D 20 (1979) 2619;

E. Gildener, Phys. Rev. D 14 (1976) 1667;

E. Gildener and S. Weinberg, Phys. Rev. D 15 (1976) 3333.

6. L. Maiani, Proceedings of the Summer School of Gif-Sur-Yvette (Paris 1980);

M. Veltman, Acta Phys. Polon. B 12 (1981) 437;

S. Dimopoulos and S. Raby, Nucl. Phys. B 192 (1981) 353;

E. Witten, Nucl. Phys. B 188 (1981) 513;

M. Dine, W. Fischler, and M. Srednicki, Nucl. Phys. B 189 (1981) 575; ibid., B 202 (1982) 238.

7. Excellent early analyses are: P. Langacker and N. Polonsky, Phys. Rev. D 47 (1993) 4028;

ibid., D 49 (1994) 1454;

L.J. Hall and U. Sarid, Phys. Rev. Lett. 70 (1993) 2673.

8. The Second Workshop on Grand Unification, University of Michigan, Ann Arbor, April 2426, 1981, eds. J.Leveille, L.Sulak, D.Unger; Birkhauser, 1981.

9. S. Weinberg, Phys. Rev. D 13 (1976) 974; D 19 (1979) 1277;

L. Susskind, Phys. Rev. D 20 (1979) 2619.

10. S. Dimopoulos and L. Susskind, Nucl. Phys. B 155 (1979) 237 ;

E. Eichten and K. Lane, Phys. Lett. B 90 (1980) 125.

11. S. Dimopoulos, S. Raby and P. Sikivie, Nucl. Phys. B 219, (1982) 479;

S. Dimopoulos and J. Ellis, Nucl. Phys. B 182 (1981) 505.

12. P. Fayet, Phys. Lett. B 69 (1977) 489; B 84 (1979) 416.

13. S. Ferrara, L. Girardello, and F. Palumbo, Phys. Rev. D 20 (1979) 403.

14. S. Dimopoulos, S. Raby, and F. Wilczek, Phys. Rev. D 24 (1981) 1681.

15. L. Girardello and M.T. Grisaru, Nucl. Phys. B 194 (1982) 65.

16. N. Sakai, Zeit.Phys. C 11 (1981) 153;

L. Ibañez and G.G. Ross, Phys. Lett. B 105 (1981) 439;

M. B. Einhorn and D. R. T. Jones, Nucl. Phys. B 196 (1982) 475;

W. J. Marciano and G. Senjanovic, Phys. Rev. D 25 (1982) 3092.

17. S. Weinberg, Phys. Rev. D 26 (1982) 287; N. Sakai and T. Yanagida, Nucl. Phys. B 197 (1982) 533.

18. S. Dimopoulos, S. Raby, and F. Wilczek, Phys. Lett. B 112 (1982) 133;

J. Ellis, D.V. Nanopoulos, and S. Rudaz, Nucl. Phys. B 202 (1982) 43.

19. S. Dimopoulos and F. Wilczek, Santa Barbara preprint, July 1981; Proceedings Erice Summer School, Ed. A. Zichichi (1981).

20. B. Grinstein, Nucl. Phys. B 206 (1982) 387; 
R.N. Cahn, I. Hinchliffe, and L. Hall, Phys. Lett. B 109 (1982) 426;

A. Masiero, D.V. Nanopoulos, K. Tamvakis, and T. Yanagida, Phys. Lett. B 115 (1982) 380 ;

K.S. Babu and S.M. Barr, Phys. Rev D 48 (1993) 5354;

D 50 (1994) 3529.

21. M. Dine, W. Fischler, Nucl. Phys. B 204 (1982) 346;

S. Dimopoulos and S. Raby, Nucl. Phys. B 219 (1982) 479;

J. Polchinski and L. Susskind, Phys. Rev. D 26 (1982) 3661.

22. E. Witten, Phys. Lett. B 105 (1981) 267.

23. E. Cremmer, S. Ferrara, L. Girardello, and A. Van Proyen, Phys. Lett. B 116 (1982) 231;

A. Chamseddine, R. Arnowitt, and P. Nath, Phys. Rev. Lett. 49 (1982) 970;

R. Barbieri, S. Ferrara, and C. Savoy, Phys. Lett. B 110 (1982) 343;

L. J. Hall, J. Lykken, and S. Weinberg, Phys. Rev. D 27 (1983) 2359.

24. L.E. Ibañez and G.G. Ross, Phys. Lett. B 110B (1982) 215;

L. Alvarez-Gaumé, M. Claudson, and M.B. Wise, Nucl. Phys. B 207 (1982) 96;

M. Dine and W. Fischler, Nucl. Phys. B 204 (1982) 346;

K. Inoue, A. Kakuto, H. Komatsu, and S. Takeshita, Prog. Theor. Phys. 68 (1982) 927 and 71 (1984) 413;

J. Ellis, D.V. Nanopoulos, and K. Tamvakis, Phys. Lett. B 121 (1983) 123;

L. Alvarez-Gaumé, J. Polchinski, and M. Wise, Nucl. Phys. B 221 (1983) 495;

L.E. Ibañez and C. Lopez, Phys. Lett. B 126 (1983) 54;

Nucl. Phys. B 233 (1984) 511;

C. Kounnas, A.B. Lahanas, D.V. Nanopoulos, and M. Quiros, Nucl. Phys. B 236 (1984) 438;

L.E. Ibañez, C. Lopez, and C. Munoz, Nucl. Phys. B 256 (1985) 218;

G. Gamberini, G. Ridolfi, and F. Zwirner, Nucl. Phys. B 331 (1990) 331.

25. S. Dimopoulos, "Supersymmetric Unification", Talk given at International Conference on the History of Original Ideas and Basic Discoveries in Particle Physics, Erice, Italy, 29 Jul - 4 Aug 1994. e-Print Archive: hep$\mathrm{ph} / 9412297$.

26. M. Carena, S. Pokorski, and C.E.M. Wagner, Nucl. Phys. B 406 (1993) 59.

27. P.H. Frampton and S.L. Glashow, Phys. Lett. B 131 (1983) 340, Erratum B 135 (1984) 515; A. Giveon, L.J. Hall, and U. Sarid, Phys. Lett B 271 (1991) 138.

28. S. Dimopoulos, S. Raby, and F. Wilczek, Phys. Today 44 (1991) 25-33.

29. N. Arkani-Hamed, S. Dimopoulos and G. Dvali, Phys. Lett. B 429, 263 (1998).

I. Antoniadis, N. Arkani-Hamed, S. Dimopoulos and G. Dvali, Phys. Lett. B 436, 257 (1998).

N. Arkani-Hamed, S. Dimopoulos and G. Dvali, Phys. Rev. D 59, 086004 (1999).

30. R. Barbieri, G. Dvali, and A. Strumia, Pisa preprint: IFUP-PTH-94-22;

31. P. Horava and E. Witten, Nucl. Phys. B 460, 506 (1996).

32. E.W. Kolb, M.S. Turner (Fermilab, Chicago U., EFI). 1990. The Early Universe. Redwood City, USA: Addison-Wesley (1990) 547 p. (Frontiers in physics 69).

33. M. Gell-Mann, P. Ramond and R. Slansky, Gauge Theories," Rev. Mod. Phys. 50, 721 (1978).

T. Yanagida, Prog. Theor. Phys. 64, 1103 (1980).

34. N. Arkani-Hamed, S. Dimopoulos, G. Dvali and J. March-Russell, hep-ph/9811448,

35. A. J. Buras, J. Ellis, M. K. Gaillard and D. V. Nanopoulos, Interactions," Nucl. Phys. B 135, 66 (1978).

36. J. Ellis and M. K. Gaillard, Phys. Lett. B 88, 315 (1979).

37. L.J. Hall, V. A. Kostelecky, and S. Raby, Nucl. Phys. B 267 (1986) 415.

H.Georgi, Phys. Lett. B 169 (1986) 231.

38. M. Dine and A. E. Nelson, Phys. Rev. D 48, 1277 (1993).

M. Dine, A. E. Nelson and Y. Shirman, Phys. Rev. D 51, 1362 (1995).

M. Dine, A. E. Nelson, Y. Nir and Y. Shirman, Phys. Rev. D 53, 2658 (1996). 
S. Dimopoulos, M. Dine, S. Raby and S. Thomas, Phys. Rev. Lett. 76, 3494 (1996).

G. F. Giudice and R. Rattazzi, Phys. Rept. 322, 419 (1999).

39. S. Dimopoulos and D. Sutter, Nucl. Phys. B 452, 496 (1995). 